\title{
\begin{tabular}{|l|l|l|l|l|}
\hline $\mathrm{M}$ & $\mathrm{R}$ & $\mathrm{S}$ & Internet Journal of & Nitride Semiconductor Research \\
\hline
\end{tabular}
}

Volume 1, Article 22

\section{Evidence for Shallow Acceptor Levels in MBE Grown GaN}

\author{
B. G. Ren \\ Department of Electrical and Electronic Engineering, University of Nottingham \\ and \\ Department of Physics, University of Nottingham \\ J. W. Orton \\ Department of Electrical and Electronic Engineering, University of Nottingham \\ T. S. Cheng \\ Department of Physics, University of Nottingham \\ D. J. Dewsnip, D. E. Lacklison \\ Department of Electrical and Electronic Engineering, University of Nottingham \\ C. T. Foxon \\ Department of Physics, University of Nottingham \\ C. H. Malloy, X. Chen \\ Department of Physics and Astronomy, University of Wales
}

This article was received on June 3, 1996 and accepted on October 24, 1996.

\begin{abstract}
We report the results of photoluminescence measurements on a number of $\mathrm{GaN}$ thin films grown by MBE on GaAs (111)B substrates. In particular, we draw attention to a new observation of a line at approximately $3.40 \mathrm{eV}$ which is accompanied by complex fine structure and interpret it as due to a donor-acceptor (DA) transition. Assuming a donor energy of $30 \mathrm{meV}$, we derive an acceptor binding energy of approximately $80 \mathrm{meV}$ which is very much smaller than the accepted value of $250 \mathrm{meV}$ for the well established $\mathrm{Mg}$ acceptor. However, our result is in agreement with a recent estimate of the hydrogenic acceptor energy as being $85 \mathrm{meV}$.
\end{abstract}

\section{Introduction}

The importance of $\mathrm{GaN}$ for practical optoelectronic devices made a quantum leap forward with the demonstration of successful p-type doping with Mg acceptors [1]. As is now well known, this led to the development of highly efficient visible LEDs [2] and more recently to the first realisation of injection laser diodes, operating at room temperature [3]. As a result, the AIGalnN material system is now seen as a major challenger to the wide gap II-VI compounds in the race to produce a viable short wavelength laser for optical disc read-out systems. However, it is interesting to note that a serious problem for both materials concerns the large ionisation energies of the commonly used acceptors. This gives rise to poor doping efficiency and correspondingly large series resistance in devices. In the case of GaN, $\mathrm{Mg}$ acceptors are characterised by a ground state some $250 \mathrm{meV}$ above the valence band edge [4] [5] which obliges device engineers to use very high doping levels in an attempt to achieve adequate free hole densities. The discovery of a shallower acceptor would, therefore, be of considerable practical importance.

On the other hand, from the scientific viewpoint, it is also desirable to understand the origin of the observed Mg level, which is considerably deeper than might reasonably be expected on the basis of the hydrogen model. The so-called chemical shifts are clearly of importance in determining acceptor binding energies and Strite [6] has drawn attention to the significance of acceptor atom d-states which can interact with Ga core electrons to influence the 
binding energy of acceptors in GaN. This is probably a major factor for the heavier acceptor atoms, $\mathrm{Zn}, \mathrm{Cd}$ and $\mathrm{Hg}$ but is not relevant for Be and $\mathrm{Mg}$ which have no electrons in the d shell. Nevertheless, the $250 \mathrm{meV}$ measured for the $\mathrm{Mg}$ level is some three-to-four times larger than one calculates on the simple hydrogen model, using the best available value for hole effective mass [7]. Can we reasonably anticipate such a large chemical shift? Or should we question the model?

One of the present authors [4] has proposed a way round the difficulty, following a suggestion of Ridley [8] that, if the binding energy is larger than the TO phonon energy (as is the case here), one should use the high frequency dielectric constant in the calculation, rather than the more usual low frequency value. This brings the calculation into line with the measured value of activation energy. However, a recent letter from Podor [5] makes a case for relating the activation energies to Phillips' electronegativities, a procedure which suggests the very different value of $85 \mathrm{meV}$ for the hydrogenic ionisation energy. There is a need for further experimental evidence as well as for clarification of the theoretical position.

The purpose of this paper is, therefore, to describe new experimental evidence concerning acceptor ionisation energies in GaN epitaxial films, evidence which tends to support the much smaller value of hydrogenic energy proposed by Podor.

\section{Experimental}

The GaN films used in our experiments were grown on (111)B GaAs substrates by a modified MBE process in which the nitrogen is supplied from an RF plasma source (Oxford Applied Research) while other species are obtained from conventional solid sources. Growth temperatures were close to $680^{\circ} \mathrm{C}$, the films were about $1 \mu \mathrm{m}$ thick and were nominally undoped. They were hexagonal in structure as demonstrated on an X-ray powder diffractometer. Photoluminescence spectra were excited with the $325 \mathrm{~nm}$ line of a Kimon He-Cd laser, dispersed by a $0.75 \mathrm{~m}$ Spex monochromator and detected with a bi-alkali photomultiplier. Laser power was typically about $5 \mathrm{~mW}$, focussed down to provide a power density of $1 \mathrm{~kW} / \mathrm{cm}^{2}$, but could be reduced by up to a thousand times using neutral density filters. Sample temperatures could be varied between $5 \mathrm{~K}$ and room temperature. Photoluminescence decay measurements were made using a frequency doubled Ti-Saphire laser pumped with a mode-locked argon ion laser. The time resolution was in the region of $60 \mathrm{ps}$.

\section{Results and Discussion}

Low temperature photoluminescence spectra in most of our samples tend to be dominated by the donor-bound exciton $\left(D^{0} \mathrm{X}\right.$ ) line at about $3.47 \mathrm{eV}$ [9] (some minor variation in position results from residual strain effects) and this line is also fairly strong in the samples referred to here. However, we also observe a relatively strong line at energies close to $3.40 \mathrm{eV}$ which we have not seen previously. An example is shown in figure 1 which covers the range of photon energies from 3.2 to $3.5 \mathrm{eV}$ and reveals a third strong line at $3.265 \mathrm{ev}$ which we believe may be due to a $\mathrm{D}^{0} \mathrm{X}$ transition from cubic $\mathrm{GaN}$ inclusions occurring randomly within the predominantly hexagonal material [10]. The other feature to which we wish to draw attention is the appearance of fine structure on the $3.40 \mathrm{eV}$ line, which actually extends over the energy range from about 3.25 to $3.47 \mathrm{eV}$, though it appears most clearly on the $3.40 \mathrm{eV}$ line itself. This structure is reproducible and can be seen more clearly in figure 2 which represents an expanded version of the same spectral region. Notice that the lower energy $(3.265 \mathrm{eV})$ line shows no sign of structure, which tends to refute any suggestion that it might be due to noise in the measurement. The individual components are spaced rather less than $1 \mathrm{mev}$ apart which is consistent with their being due to discrete donor-acceptor pair lines in an overall spectrum in which the $3.40 \mathrm{eV}$ line represents the contribution from distant pairs (see, for example, the much earlier observations on CdS reported by Henry et al [11]).

The significance of this interpretation lies in the implication for the energy of the acceptor involved. Using the well known expression for the DA photon energy:

$$
h=E_{g}-\left(E_{A}+E_{D}\right)+e^{2} / 4{ }_{0} R
$$

taking the donor energy $E_{D}=30 \mathrm{meV}$ and assuming a value of $10 \mathrm{meV}$ for the Coulomb term appropriate to distant pairs (in line with ref 11), we obtain an accceptor energy $E_{A}=80 \mathrm{meV}$ which is in excellent agreement with that predicted for the hydrogenic acceptor by Podor.

This novel result clearly requires justifying. In the absence of supporting evidence, the $3.40 \mathrm{eV}$ line could obviously

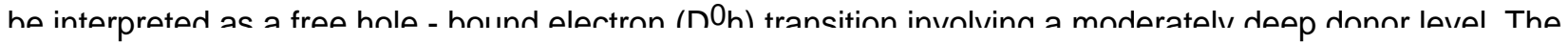


discrete pair lines provide the strongest justification but they are not well resolved and cannot be interpreted in detail (as was also true in CdS) so we look for other evidence to support our interpretation. We have performed two further experiments, examining the way in which the $3.40 \mathrm{eV}$ line depends on temperature and on excitation intensity. In figure 3 we compare the temperature dependence of the $3.40 \mathrm{eV}$ line with that of the $\mathrm{D}^{0} \mathrm{X}$ line and of the band gap. The latter tends initially to move slightly closer to the band edge (due to exciton delocalisation) then moves with the band edge, as expected. Over the temperature range for which it can be followed, the $3.40 \mathrm{ev}$ line moves more or less steadily towards the band edge, similar to the behaviour of the well established DA transitions which appear at 3.267eV in many GaN samples [12], the rate of approach being roughly $2 \mathrm{kT}$. Free-to-bound transitions also move closer to the band edge with increasing temperature (due to the increasing kinetic energy of the free particle) but at the much slower rate, given by kT/2 [13].

Another feature of DA transitions is their tendency to move towards higher photon energies as the excitation density is increased (due to saturation of the longer lived more distant pairs). This is in accord with our measurements on the $3.40 \mathrm{ev}$ line. Reducing the excitation density by a factor of a hundred shifted the peak position downwards by $15 \mathrm{meV}$, while broadening the line from $21 \mathrm{meV}$ to $55 \mathrm{meV}$. (The $\mathrm{D}^{0} \mathrm{X}$ line remained sensibly unchanged). For completeness, we should also note the possibilty of free-to-bound transitions shifting upwards with increasing excitation power, as a result of band filling but an order-of-magnitude calculation suggests such a shift as we observe to be highly unlikely for the free-to-bound case. More significant, perhaps, is the observation that band filling should result in line broadening at high excitation levels, rather than the opposite effect which we find experimentally. Finally, we note that preliminary measurements of PL decay made in Cardiff show evidence of very long decay times ( order of several microseconds ) which is also characteristic of DA pair recombination.

We can only speculate about the origin of the acceptor level involved. It is widely accepted that carbon is an impurity to be found in most MBE machines and there is no reason to believe anything different in our case. Therefore we suggest carbon as being the most likely acceptor, though we are unclear why it should be prominent in samples grown recently, when not previously recognised over three years of nitride growth.

\section{Conclusions}

We have studied the photoluminescence spectra of a number of samples of GaN grown by MBE on GaAs (111)B substrates and found a hitherto unknown emission line at a photon energy near $3.4 \mathrm{eV}$. This line is frequently observed to be associated with a complex fine structure which we interpret as arising from discrete donor-aceptor pair lines, the broader $3.4 \mathrm{eV}$ line corresponding to distant pairs. Confirmation of the DA nature of the line is provided by measurement of its temperature and excitation density dependence. Taking the donor energy to be $30 \mathrm{meV}$ results in a value of approximately $80 \mathrm{meV}$ for the acceptor (which we tentatively attribute to carbon impurities). This is very significantly smaller than the well established value of $250 \mathrm{meV}$ associated with the $\mathrm{Mg}$ acceptor in GaN and suggests the possibility of shallower acceptor levels than were previously thought possible. It also supports a recent sugestion that the hydrogenic acceptor energy in GaN is $85 \mathrm{meV}$.

\section{Acknowledgments}

B G Ren would like to thank The Royal Society for financial support for his visit to Nottingham.

The work was supported under ESPRIT project 20968, LAQUANI.

\section{References}

[1] H. Amano, M. Kito, K. Hiramatsu, I. Akasaki, Jpn. J. Appl. Phys. 28, L2112 (1989).

[2] Shuji Nakamura, Takashi Mukai, Masayuki Senoh, Appl. Phys. Lett. 64, 1687-1689 (1994).

[3] S Nakamura, M Senoh, S Nagahama, N Iwasa, T Yamada, T Matsushita, H Kiyoku, Y Sugimoto, Jpn. J. Appl. Phys. 35, L74-L76 (1996).

[4] J W Orton, Semicond. Sci. Technol. 10, 101 (1995).

[5] B Podor, Semicond. Sci. Technol. 11, 827 (1996).

[6] S Strite, Jpn. J. Appl. Phys. 33, L699-L701 (1994). 
[7] C. Merz, M. Kunzer, U. Kaufmann, Semicond. Sci. Technol. 11, 712 (1996).

[8]B. K. Ridley, Quantum Processes in Semiconductors, (Oxford University Press, 1993), p62.

[9] R. Dingle, D. D. Sell, S. E. Stokowski, M. Ilegems, Phys. Rev. B 4, 1211 (1971).

[10] A V Andrianov, D E Lacklison, J W Orton, D J Dewsnip, S E Hooper, C T Foxon, Semicond. Sci. Technol. 11, 366 (1996).

[11] C H Henry, R A Faulkner, K Nassau, Phys. Rev. 183, 798 (1969).

[12] O. Lagerstedt, B. Monemar, J. Appl. Phys. 45, 2266 (1974).

[13] D M Eagles, J. Phys. Chem. Sol. 16, 76 (1960).

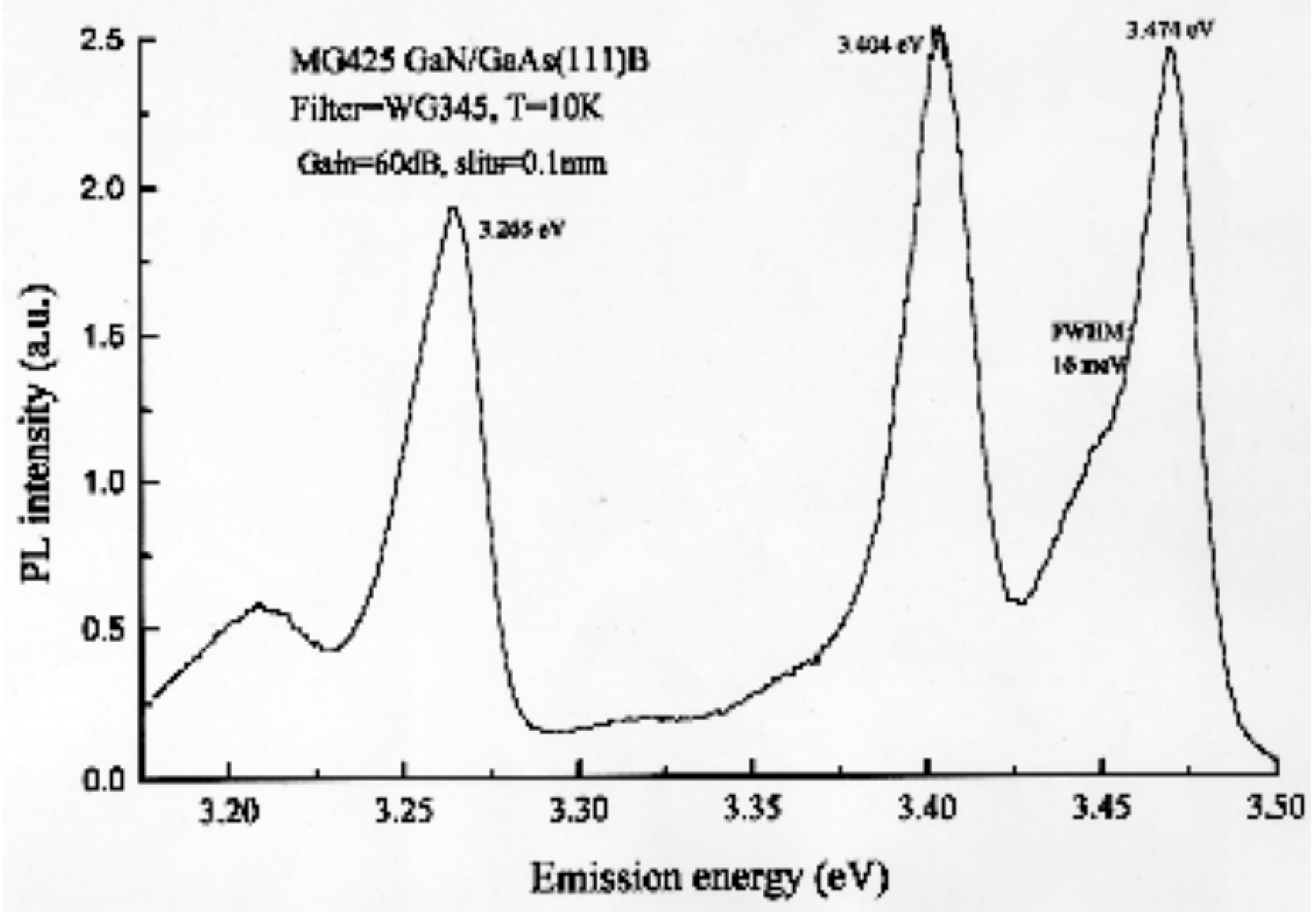

Figure 1. Photoluminescence spectrum of sample $M G 425$, showing the $D^{0} X$ transition at $3.474 \mathrm{eV}$, the new DA line at $3.404 \mathrm{eV}$ and a third line at $3.265 \mathrm{ev}$ which we attribute to a $\mathrm{D}^{0} \mathrm{X}$ transition in cubic GaN inclusions. Note the structure on top of the DA line which extends on either side but does not appear on the $3.265 \mathrm{eV}$ line. 


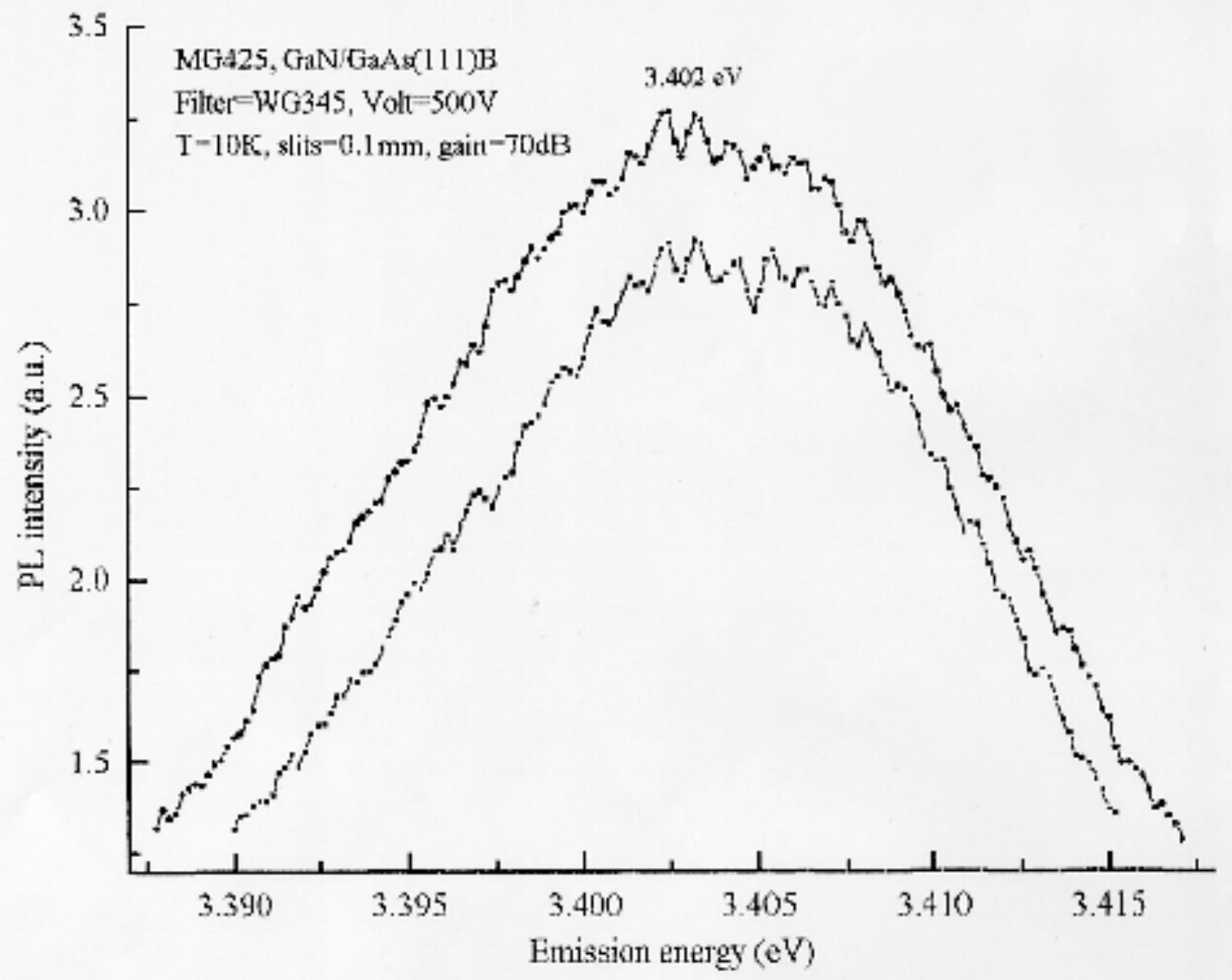

Figure 2. Detail of the DA line at $3.40 \mathrm{ev}$, showing the fine structure with greater clarity. The two curves represent two distinct scans, showing good reproducibility in the structure. 


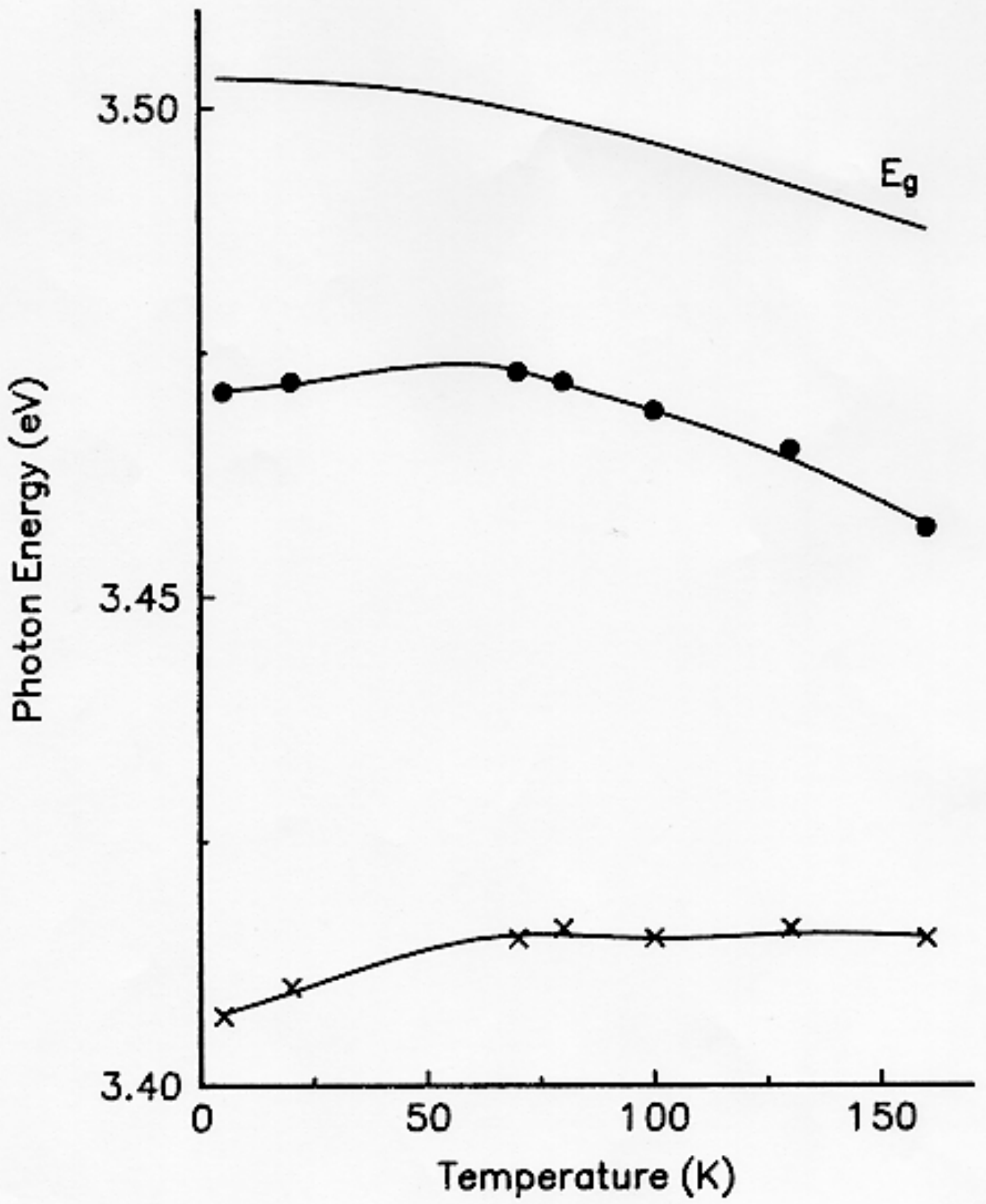

Figure 3. Plots of the temperature dependence of the photon energies of the $D^{0} X$ and $D A$ transitions, compared with that of the GaN band edge. The DA line does not follow the band edge but moves closer to it as the temperature increases, in a similar manner to that of other DA lines in GaN.

(C) 1996-1997 The Materials Research Society

\begin{tabular}{|l|l|l|l|l|}
\hline $\mathrm{M}$ & $\mathrm{R}$ & $\mathrm{S}$ & Internet Journal of & Nitride Semiconductor Research \\
\hline
\end{tabular}

